\title{
Bouncing jet: A Newtonian liquid rebounding off a free surface
}

\author{
Matthew Thrasher, ${ }^{*}$ Sunghwan Jung, ${ }^{\dagger}$ Yee Kwong Pang, ${ }^{\ddagger}$ Chih-Piao Chuu, and Harry L. Swinney ${ }^{\S}$ \\ Center for Nonlinear Dynamics and Department of Physics, University of Texas at Austin, Austin, Texas 78712, USA
}

(Received 11 July 2007; published 29 November 2007)

\begin{abstract}
We find that a liquid jet can bounce off a bath of the same liquid if the bath is moving horizontally with respect to the jet. Previous observations of jets rebounding off a bath (e.g., the Kaye effect) have been reported only for non-Newtonian fluids, while we observe bouncing jets in a variety of Newtonian fluids, including mineral oil poured by hand. A thin layer of air separates the bouncing jet from the bath, and the relative motion replenishes the film of air. Jets with one or two bounces are stable for a range of viscosity, jet flow rate and velocity, and bath velocity. The bouncing phenomenon exhibits hysteresis and multiple steady states.
\end{abstract}

DOI: 10.1103/PhysRevE.76.056319

PACS number(s): 47.15.Uv, 47.55.Ca, 47.85.mf, 47.20.Gv

\section{INTRODUCTION}

A liquid stream falling onto the free surface of a liquid bath can merge immediately on contact, plunge through the surface and entrain air [1,2], coil up like a rope [3], float on the surface prior to coalescing [4], float without ever coalescing [5], or break into droplets [6]. We report in this paper observations of a jet of Newtonian liquid bouncing off a horizontally moving surface of a bath of the same liquid. Figure 1 shows a typical bouncing jet viewed from the side. The jet falls to the bath's surface, is bent upward, and undergoes a short flight. After rebounding once more off the surface, the stream merges with the bath. In all figures the liquid bath is moving to the right, and the stream and bath are the same fluid. This paper examines when and how a liquid jet bounces. The issues that arise in studying the bouncing jet (e.g., noncoalescence, lubrication, and entrainment) are ubiquitous in fluid processing, such as pouring and mold casting. They also are critical in the design of bearings [7], gas-liquid reactors [8], film coating equipment [9], and metallurgical procedures [6].

Drops of liquid floating and bouncing on the surface of a bath have been studied scientifically for over 125 years [10-13]. On a pond during a light rainfall, splashing raindrops throw up smaller drops, and these smaller drops often can be seen to sit on the water surface momentarily. During this time of noncoalescence, a thin layer of air separates each drop from the pond. Noncoalescence can be prolonged (sometimes indefinitely) by either replenishing the air between the two liquid bodies or slowing the loss of the existing air. This can be achieved with surfactants $[14,15]$, vibration [4], microgravity [16], a velocity difference between the drop and bath [7], evaporation [17], thermocapillarity [17], or by increasing the viscosity of the surrounding medium [18].

The bouncing of a liquid jet has also been observed for a fluid of elongating polymers incident at a glancing angle on

\footnotetext{
*thrasher@chaos.utexas.edu

Present address: Department of Mathematics, MIT, Cambridge, MA 02139 USA.

${ }^{\ddagger}$ Present address: The Hong Kong University of Science and Technology, Clear Water Bay, Kowloon, Hong Kong.

§swinney@chaos.utexas.edu
}

a rotating drum [19]. Bouncing also occurs for a jet of shearthinning liquid falling onto a pool of the same liquid; this is called the Kaye effect [20]. While this effect is visually similar to the bouncing jet phenomenon presented in this paper, the Kaye effect occurs only in non-Newtonian fluids [21] (see discussion in Sec. VI).

Jets of water colliding midair at a glancing angle can also bounce off each other, because during the collision they are separated by a layer of air [5,22]. However, no systematic study has been conducted of Newtonian jets bouncing off a bath surface. To measure the conditions necessary for the bouncing of a Newtonian liquid jet, we built an experimental apparatus (Sec. II), and we found that jets bounce for a wide range of parameters (Sec. III). By comparing the energies associated with the nonbouncing and bouncing states, we suggest why bouncing is preferred rather than plunging (Sec. IV). A bouncing jet can easily be reproduced with common materials (Sec. V). These observations are related to previous work in Sec. VI.

\section{EXPERIMENT}

We used a rotating annulus of fluid (Fig. 2) to maintain a constant horizontal velocity of a bath with respect to a ver-

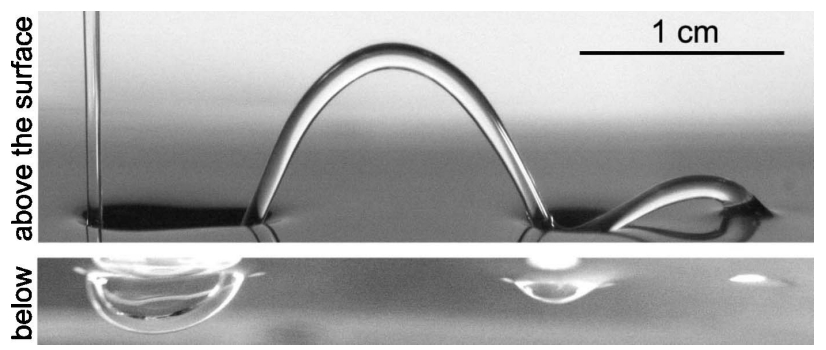

FIG. 1. A liquid jet bounces twice before merging with the bath, which is moving to the right. The jet and bath are silicone oil of the same viscosity. The upper and lower pictures were taken from above and from below the bath surface; the images were not obtained at the same time or the same angle, so small differences exist. The jet's image can be seen reflected on the surface. Parameters: liquid viscosity $\mu=102 \mathrm{mPa}$ (about 100 times more viscous than water), jet flow rate $Q=0.35 \mathrm{~cm}^{3} / \mathrm{s}$, falling height $H$ $=5.0 \mathrm{~cm}$, and horizontal velocity of the bath $V_{\text {bath }}=15.7 \mathrm{~cm} / \mathrm{s}$. 


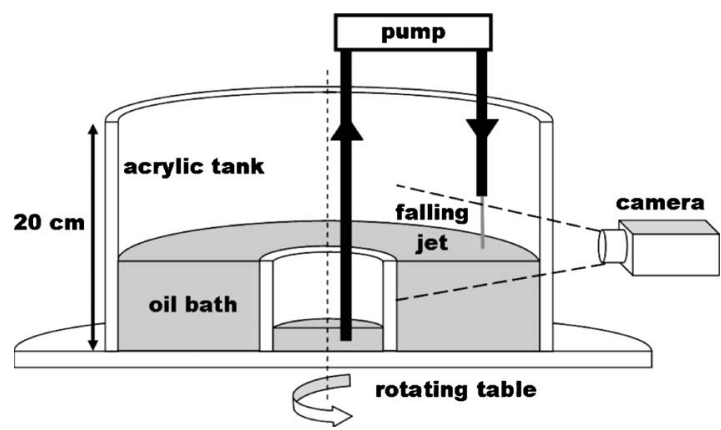

FIG. 2. Experimental setup: a bath of silicone oil is rotated under a falling stream of the same oil. A camera in the laboratory frame records the motion through the tank's clear acrylic side.

tically impinging jet. The parameters varied were the viscosity $\mu$ of the silicone oils, the jet's flow rate $Q$, the height of the nozzle $H$ above the bath surface, and the relative velocity $V_{\text {bath }}$ of the bath to the nozzle.

A cylindrical tank with a clear acrylic outer wall was mounted on a rotating table. The annular bath was $39.1 \mathrm{~cm}$ in outer diameter, $27.3 \mathrm{~cm}$ in inner diameter, and $7.7 \mathrm{~cm}$ deep. Silicone oils were used for their stability, low surface tension, high viscosity, and Newtonian properties. They were Dow Corning 200 series and Clearco oils with viscosity $\mu$ $=52-349 \mathrm{mPa} \mathrm{s}$, density $\rho=959-968 \mathrm{~kg} / \mathrm{m}^{3}$, and surface tension $\sigma=21.0-21.2 \mathrm{mN} / \mathrm{m}$. Measurements were made at the bath temperature of $23 \pm 1{ }^{\circ} \mathrm{C}$. We measured the viscosity of each oil for shear rates from 1 to $10^{4} \mathrm{~s}^{-1}$ with a Paar Physica MCR300 rheometer, and we found at most a weak dependence on shear rate: oils with viscosities of $\mu=52,102$, 211 , and $349 \mathrm{mPa} s$ at a low shear rate had viscosity values $2 \%, 4 \%, 8 \%$, and $12 \%$ lower at $10^{4} \mathrm{~s}^{-1}$, respectively.

The typical shear rate in the liquid in our experiments is difficult to estimate because the velocity profiles in the air and liquid were not measured. Most of the shear was in the air layer since the dynamic viscosity ratio of air to oil ranged from $5 \times 10^{-5}$ to $4 \times 10^{-4}$. Even ignoring the air layer, the largest velocity difference $[1.7 \mathrm{~m} / \mathrm{s}$; see Fig. 5(b) below] across the smallest jet diameter $(0.05 \mathrm{~cm})$ would produce a maximum shear rate of $3400 \mathrm{~s}^{-1}$, at which even the most viscous oil decreased in viscosity by only a few percent. The actual shear rates in the liquid phase should be much smaller; hence the silicone oils used can be considered as Newtonian fluids for the conditions in the experiment.

The table's rotation rate determined the relative velocity between the bath surface and the nozzle $\left(V_{\text {bath }} \equiv \Omega R\right.$ ranged from less than 1 to $35 \mathrm{~cm} / \mathrm{s}$ with a typical distance from the rotation axis $R=16 \mathrm{~cm}$ and a rotation rate $\Omega / 2 \pi=0-0.4 \mathrm{~Hz}$ ). When changing the rotation rate, adequate time was given for the bath to establish solid-body rotation. The typical uncertainty in the horizontal bath velocity was $1 \%$.

The flow rate $Q$ was controlled by a gear pump with a pulse dampener and a bypass; $Q$ ranged from 0.075 to $6.28 \mathrm{~cm}^{3} / \mathrm{s}$ with a typical uncertainty of $2 \%$. Excess liquid drained over an interior wall into a central reservoir from which liquid was pumped; thus the bath's surface height was constant at the inner cylinder, barring interfacial pinning. The rotation rate changed the bath level at the jet's position by at most $0.1 \mathrm{~cm}$.
The pump withdrew oil from the central basin and released it above the surface through a vertical Teflon nozzle (stationary in the laboratory frame). The nozzle had an inner diameter $d_{\text {nozzle }}=0.52 \mathrm{~cm}$ and produced a vertical liquid stream at a height $H$ above the liquid surface; $H$ ranged from 0.7 to $15 \mathrm{~cm}$, as determined (within $3 \%$ ) using a cathetometer. The velocity of the jet $V_{j e t}$, measured at the point of first impact with the surface, was mostly due to falling from height $H$ and only changed slowly with $Q$ (i.e., $\left.4 Q / \pi d_{n o z z l e}^{2} \ll \sqrt{2 g H}\right)$. With typical values $V_{\text {jet }}=60 \mathrm{~cm} / \mathrm{s}$ and the jet diameter $d_{\text {jet }}=0.1 \mathrm{~cm}$, the Reynolds number of the jet was 6 , the Bond number was 0.5 , the capillary number was 3 , and the Weber number was 18. Dust and bubbles sitting on the surface could destabilize a bouncing jet, so they were removed by dragging a mesh over the surface of the bath opposite to the falling jet (not shown in Fig. 2).

The bouncing was initiated by passing a small $(0.6 \mathrm{~cm}$ diameter) horizontal rod quickly through the falling jet, changing the radius, velocity, and shape of the jet in a complicated, time-dependent manner. When the nonuniformity collided with the bath surface, a nonbouncing jet often started to bounce.

Two other methods were found to initiate the bouncing, but were not used in mapping the regime diagrams in Sec. III B. One method was to rapidly decrease the flow rate from a high rate that entrained air. As the flow rate decreased, the submerged jet penetrated less deeply and then began to bounce. Images and a movie of this process are available in [23]. The other method was to change the bath velocity, as discussed more in Sec. III C.

Images were acquired in the laboratory frame through the outer wall of the tank with a digital camera. If an ambiguity existed in the geometry of the jet and bath, images were taken at several angles. The images were used to measure the diameter of the jet, and the velocity was computed by using the flow rate and continuity. The typical uncertainty of the vertical velocity measurement was $8 \%$.

\section{RESULTS}

\section{A. Dependence on bath velocity and flow rate}

Two important parameters that change the qualitative behavior of the jet are the bath velocity and the flow rate. At low bath velocity, the jet bounced with a nearly vertical rebound, as in Fig. 3(a). At smaller bath velocity, the jet bounced only intermittently because the bouncing liquid would collide with the falling jet or would distort the surface and destabilize the bouncing. To get a stable bounce the bath velocity had to be fast enough to carry away the rebounding fluid so it would not disturb the impinging jet. The bouncing could stop in another way: the height of the jet's bounce would quickly decrease until the jet merged with the bath.

As the bath velocity increased, the rebound became more oblique, as in Fig. 3(b). With increasing bath velocity, the jet gained more horizontal momentum from the bath and the angle of the jet's rebound changed continuously.

During bouncing, the jet and the bath were separated by a lubricating layer of air, as revealed by a laser beam propagating down the falling jet. The beam was internally reflected 

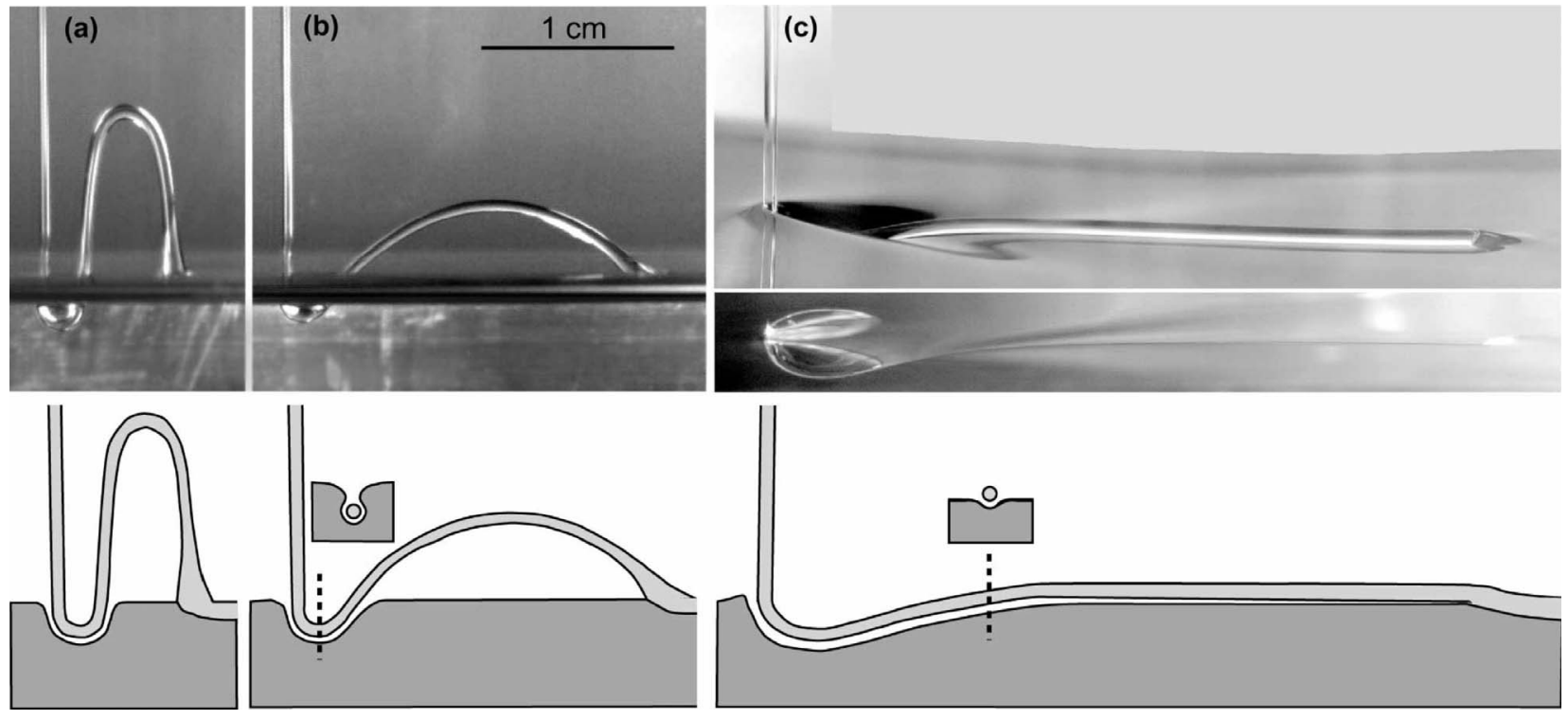

FIG. 3. Photographs (top) and schematic drawings (bottom) of the jet for different horizontal velocities of the bath, $V_{\text {bath }}$ : (a) $0.656 \mathrm{~cm} / \mathrm{s}$, a jet bouncing nearly vertically; (b) $3.28 \mathrm{~cm} / \mathrm{s}$, a jet bouncing more obliquely; (c) $42.3 \mathrm{~cm} / \mathrm{s}$, a trailing (nonbouncing) jet. Increasing $V_{\text {bath }}$ imparts more horizontal momentum to the rebounding jet. The schematics exaggerate the thickness of the layer of air between the jet and bath. For (a) and (b), the dark horizontal line is the surface's meniscus on the outer tank wall; in (c), the top and bottom images were taken above and below the surface, so that the surface extends back in perspective. Parameters in (a) and (b) were $\mu=349 \mathrm{mPa}$, $Q$ $=0.16 \mathrm{~cm}^{3} / \mathrm{s}, H=4.2 \mathrm{~cm}$; in (c), $\mu=102 \mathrm{mPa} \mathrm{s}, Q=0.35 \mathrm{~cm}^{3} / \mathrm{s}, H=5.0 \mathrm{~cm}$.

within the jet while the jet bounced, and entered the bath only when the jet and bath merged afterward. We did not measure the thickness of the air layer, but for a plunging jet the air layer surrounding a jet has been measured by Lorenceau et al. [2] to be several micrometers thick. For a rebounding water drop, the minimum thickness of the air film was calculated by Jayaratne and Mason [24] to be about $0.1 \mu \mathrm{m}$. For a pendant drop suspended above a moving solid surface, the thickness of the film was measured by Vetrano and Dell'Aversana to be a few micrometers on average [17].

The velocity of the bath necessary for a stable bounce was small compared to the jet velocity. Defining the jet incidence angle as $\theta=\tan ^{-1}\left(V_{\text {jet }} / V_{\text {bath }}\right)$, we found that $\theta$ ranged only from $83^{\circ}$ to $90^{\circ}$, while the angle of rebound (in the bath's frame) ranged from $20^{\circ}$ to $80^{\circ}$ (for $\mu=349 \mathrm{mPa} \mathrm{s}, Q$ from 0.16 to $0.52 \mathrm{~cm}^{3} / \mathrm{s}, H=4.2 \mathrm{~cm}$, and $V_{\text {bath }}$ from 0.7 to 7.9 $\mathrm{cm} / \mathrm{s}$ ). For these conditions, $13-29 \%$ of the jet's speed was lost while bouncing. Typically, the jet rebounded with higher speed with increasing $V_{\text {bath }}$ until the jet rebounded low enough for its increasing contact with the bath to slow the jet substantially. This trend is consistent with measurements made by Jayaratne and Mason of the rebound of individual water drops from a water surface [24].

At a higher bath velocity, the jet no longer lifted off the surface of the bath, but rather floated on top of the bath, as in Fig. 3(c). We call this state the "trailing jet." The trailing jet could be readily identified by looking from below the surface at the continuous indentation made by the jet on the surface. The jet did not coalesce with the bath until the thin layer of air between it and the bath drained enough to burst, or until the air layer was disturbed by dust or an irregularity in the jet. The trailing jet often collapsed in long sections at a time, which indicated that the air film had ruptured in the beginning or middle of the trailing jet.

The transition between a bouncing jet and a trailing jet was abrupt in some cases and gradual in others. For example, at $\mu=102 \mathrm{mPa}$ s and $Q$ between 0.10 and $1.05 \mathrm{~cm}^{3} / \mathrm{s}$, the jet would no longer bounce above a particular bath velocity. Instead, the jet would trail along the surface; the length of liquid floating on the surface quickly shortened until the jet merged with the bath. On the other hand, for $\mu$ $=211 \mathrm{mPa} \mathrm{s}$ and $Q$ between 0.55 and $0.94 \mathrm{~cm}^{3} / \mathrm{s}$, the jet would lift off the surface less and less as $V_{\text {bath }}$ increased, until a jet of constant length floated on the surface.

We expected that the length of the trailing jet would be correlated with the bath velocity, but we found the behavior to be more complicated, e.g., for $\mu=211 \mathrm{mPa} \mathrm{s}, Q$ $=0.55 \mathrm{~cm}^{3} / \mathrm{s}$, and $H=3.0 \mathrm{~cm}$ : when $V_{\text {bath }}$ exceeded 12.2 $\mathrm{cm} / \mathrm{s}$, the bounce trailed along the surface; for $V_{\text {bath }}$ from 12.2 to $17.9 \mathrm{~cm} / \mathrm{s}$, the trailing jet length increased; for $V_{\text {bath }}$ from 17.9 to $26.1 \mathrm{~cm} / \mathrm{s}$, the length decreased until it was only a few jet diameters long; and when $V_{\text {bath }}$ exceeded $26.1 \mathrm{~cm} / \mathrm{s}$, the length increased until it was many centimeters long. This paper concentrates on the bouncing phenomena.

The bouncing jet's behavior also depends on flow rate, as Fig. 4 illustrates. The higher the flow rate, the more vertical momentum the jet has to deform the bath's surface. This leads to a deeper, ellipsoidal indentation and more viscous drag on the jet by the bath. The decrease in jet velocity can be seen by the thickening of the jet and the smaller bounce height. The bouncing jet in Fig. 4(b) is slightly irregular. Irregularities in the pumping, nozzle position, and surrounding air can cause the bouncing jet to be unsteady. If the irregularity is temporary, the unsteady motion usually decays back to the steady bouncing jet. 

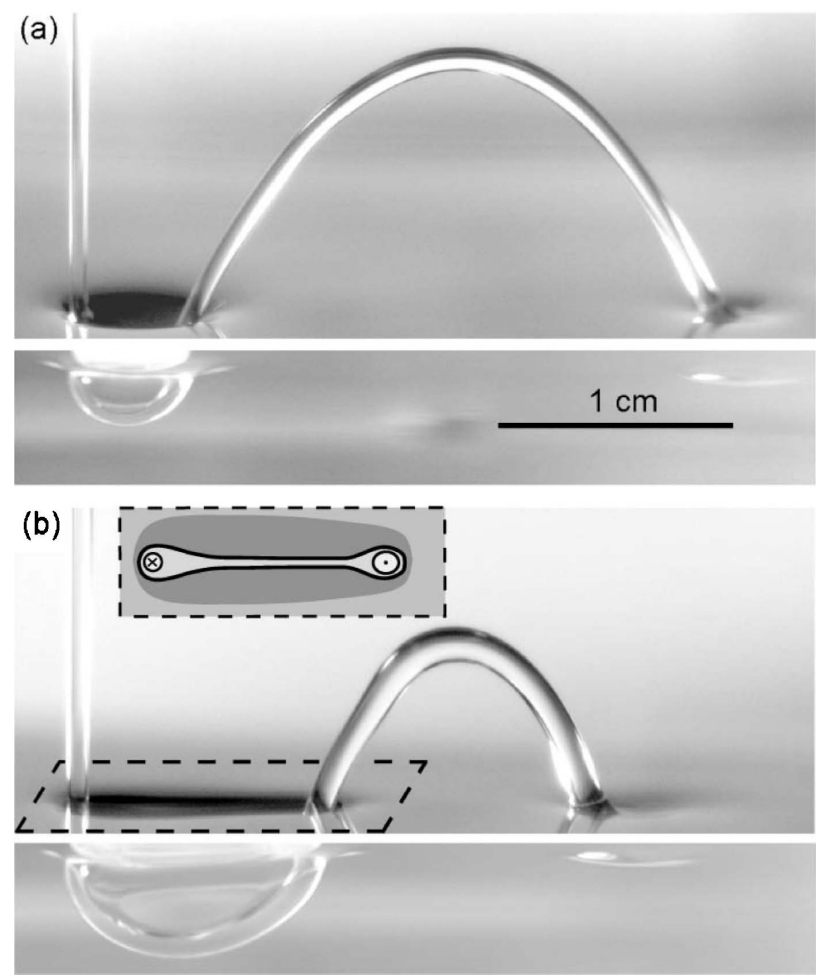

FIG. 4. Photographs of the bouncing jet for flow rates $Q$ : (a) 0.23 and (b) $0.44 \mathrm{~cm}^{3} / \mathrm{s}$; the top and bottom pictures in each case are, respectively, above and below the surface. The jet with the greater flow rate pushes the surface deeper and the jet rebounds to a lower height. Each jet rebounds moving more slowly than its initial speed (so that, by continuity, the jet becomes thicker). The inset in (b) is a top view of the area with the dashed outline; this region in the picture is just below the bath level and is in shadow. The closed black curves within the inset show a horizontal cross section of the interfaces just below the bath level. Parameters for both (a) and (b): $\mu=102 \mathrm{mPa} \mathrm{s}, H=5.0 \mathrm{~cm}, V_{\text {bath }}=15.7 \mathrm{~cm} / \mathrm{s}$.

During rebound, the jet is below the bath's surface level for some distance; the jet's weight and the changing momentum of the jet are balanced by surface tension and buoyancy [see the inset of the schematic in Fig. 3(b)]. However, for most jets, the buoyancy of the indentation is small because the sides of the surface indentation are close together and little volume is displaced [see the inset of Fig. 4(b)]. Also the jet's weight can be neglected relative to the large change in the jet's momentum. In this case the force changing the jet's momentum is provided mainly by surface tension. The surface pulls nearly vertically on the length $\ell$ of the jet that is under the bath's surface, producing a force $F_{S} \approx 2 \sigma \ell$. Assuming that the jet velocity is the same before and after rebound, the rate of change of the jet's vertical momentum is $F_{I}$ $=\Delta p_{y} / \Delta t_{c} \approx \rho \pi\left(d_{j e t} / 2\right)^{2} V_{j e t}^{2}(1+\sin \phi)$, where $\Delta p_{y}$ is the change in the jet's momentum in the vertical direction, $\Delta t_{c}$ is the duration of the collision, and $\phi$ is the angle of rebound measured from the horizon. In Fig. 4(b), the length $\ell$ $=1.3 \pm 0.1 \mathrm{~cm}$ and the angle $\phi=(70 \pm 2)^{\circ}$. Therefore, for this simplified force calculation, the surface force $F_{S}$ $=0.54 \pm 0.04 \mathrm{mN}$ and the force of the jet changing direction $F_{I}=0.52 \pm 0.06 \mathrm{mN}$, where only the measurement uncertain-
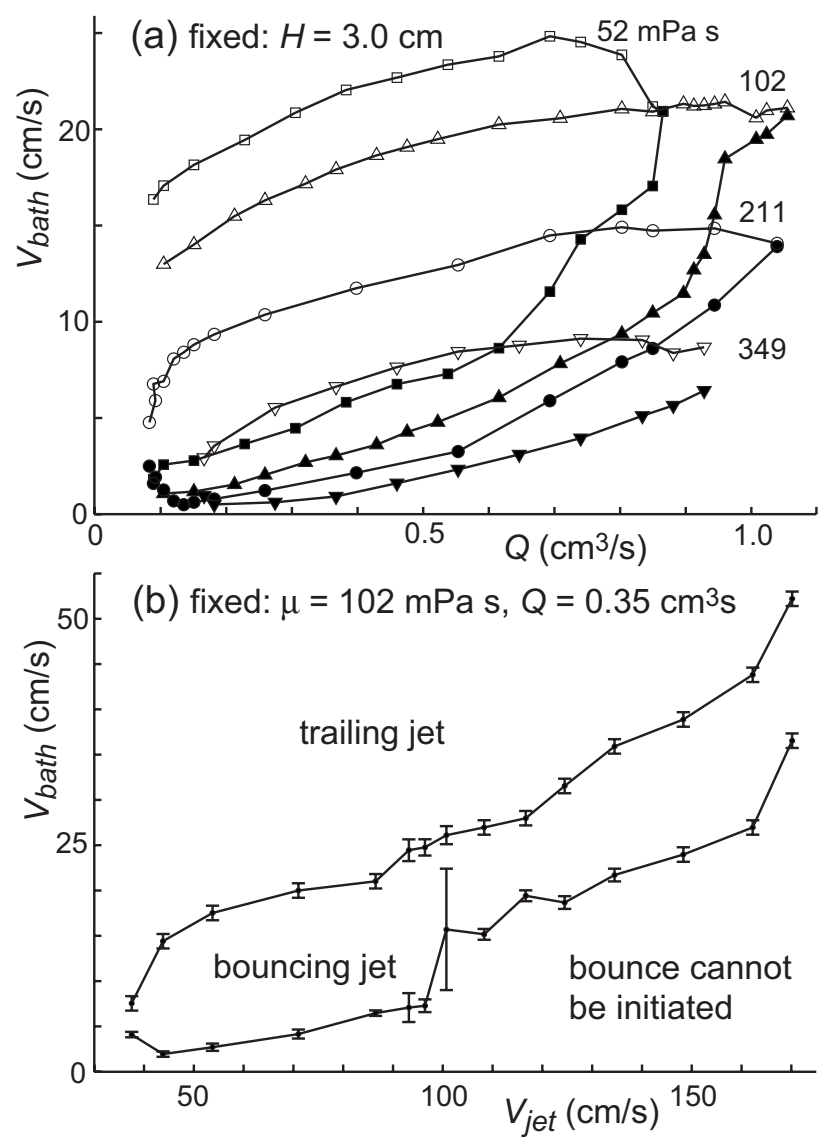

FIG. 5. Conditions for which the jet bounces. (a) Bounces could be initiated between the lines with closed and open points but could not be initiated below the lines with closed points or above the open points; a trailing jet was often observed above the open points [viscosity values $\mu=53(\boldsymbol{\square}, \square), 102(\boldsymbol{\Delta}, \triangle), 211(\bullet, \bigcirc)$, and 349 $\mathrm{mPa} \mathrm{s}(\boldsymbol{\nabla}, \nabla)]$. The bath velocity at which the transitions occur was reproducible typically within $4 \%$; the uncertainty was greater where the transition curves have a steeper slope. (b) Dependence of the region of stable bounce on $V_{\text {jet }}$ and $V_{\text {bath }}$; the range of $V_{\text {jet }}$ (38 $-170 \mathrm{~cm} / \mathrm{s}$ ) corresponds to $H$ values $1.7-14.1 \mathrm{~cm}$. Error bars show the uncertainty of each transition point. Around $V_{\text {jet }} \approx 100 \mathrm{~cm} / \mathrm{s}$, there is a greater uncertainty corresponding to a transition from a nonentraining jet to an air-entraining jet as $V_{j e t}$ is increased.

ties are included here (systematic errors from the approximations made in the argument are not included). The forces are equal within the measurement uncertainty, which is much less than the uncertainty of the approximations.

\section{B. Regime diagrams}

Sweeps of the parameters $\mu, Q, H$, and $V_{\text {bath }}$ were conducted with either $H$ held constant [Fig. 5(a)] or $\mu$ and $Q$ held constant [Fig. 5(b)]. Each point was measured at least three times. Bouncing was initiated by passing a plastic rod through the falling liquid stream. A bouncing stream was considered "initiated" after $5 \mathrm{~s}$; most jets that were stable for $5 \mathrm{~s}$ would persist for much longer times. The initiation procedure yielded transition parameter values that were reproducible and consistent for different experimenters. The 

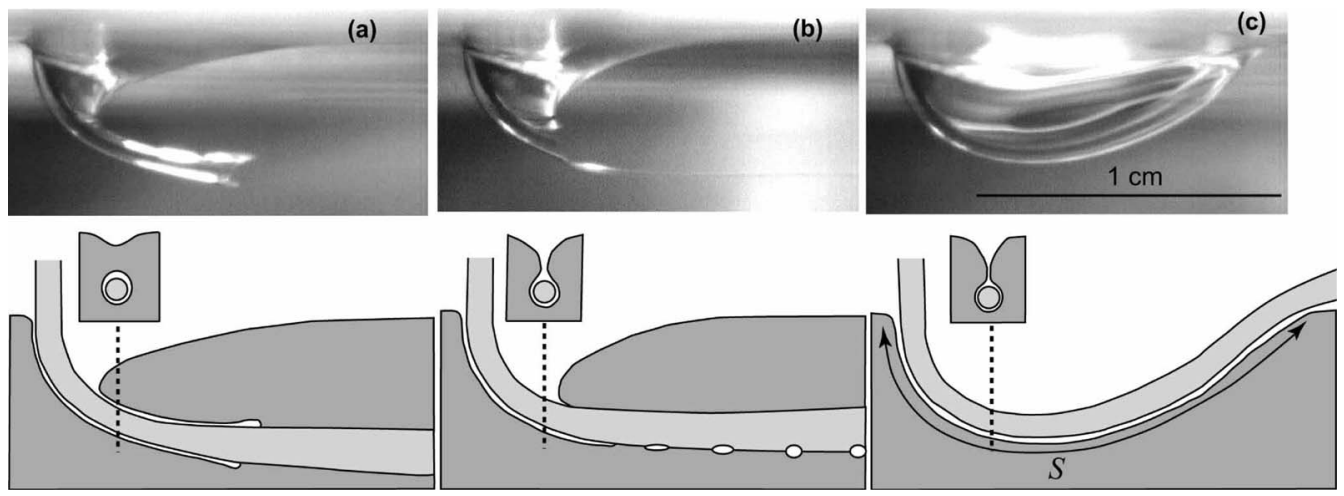

FIG. 6. Multiplicity: the three states (a)-(c) are stable for the same conditions. The bath surface is at the top of each photograph, and below each photograph is a corresponding schematic diagram. (a) Plunging jet with air entrainment and no bounce; bubbles break off from the top edge of the entrained air sheath. (b) Half-entraining jet, which entrains air only on its lower edge; bubbles (blurred to a faint line by the long exposure) break off from the entrained air skirt. (c) Bouncing jet, whose curved surface refracts light and produces a distorted image of the background. Parameters: $\mu=106 \mathrm{mPa} \mathrm{s}, Q=0.35 \mathrm{~cm}^{3} / \mathrm{s}, H=6.4 \mathrm{~cm}, V_{\text {bath }}=15.2 \mathrm{~cm} / \mathrm{s}$.

choice of $5 \mathrm{~s}$ persistence for identifying a stable bounce is arbitrary; a criterion of $1 \mathrm{~s}$ duration would yield parameter space regions for bouncing somewhat larger than those in Fig. 5.

Figure 5(a) displays the range of $V_{b a t h}$ for which a bounce could be initiated as a function of $Q$ (for a fixed nozzle height, $H=3.0 \mathrm{~cm})$. For each oil viscosity value, there is a transition between no bouncing and bouncing, marked by solid points in Fig. 5(a). The open points mark the greatest bath velocity where bouncing could be initiated. Above the open points, the jet often trailed on the surface either temporarily or steadily.

The regime where bouncing could be initiated did not close at low $Q$ for the viscosities of $\mu=52$ and $102 \mathrm{mPa} \mathrm{s}$; the jet broke into droplets before reaching the surface. To prevent dripping, care was taken so that the oil did not wet the Teflon nozzle beyond the rim of its opening; however, dripping could not be prevented for low $Q$. For $\mu$ $=349 \mathrm{mPa} \mathrm{s}$ at high $Q$, the bouncing regime did not close because the jet no longer lifted off the surface for higher flow rates. At viscosities higher than $349 \mathrm{mPa} \mathrm{s}$, the transitions from nonbouncing to bouncing became difficult to reproduce because of sensitivity to mechanical vibration. At some conditions, mechanical noise kept the jet bouncing, while at other conditions, the same amount of noise destabilized the bouncing jet.

Jets can bounce twice, as in Fig. 1, but this occurs in a smaller parameter space region than the jet undergoing a single bounce (Fig. 5); the region of double bouncing was not mapped.

The region of stable bouncing when $H$ rather than $Q$ was varied is shown in Fig. 5(b). Roughly, for higher $H$ (and hence higher $V_{j e t}$ ), a higher horizontal bath velocity is needed for stable bouncing.

The range in which stable bouncing occurs is hysteretic in two senses. First, the region in which the jet bounces is larger if the experimental parameters are changed after a bounce has been initiated. For example, if the bath's velocity was decreased slowly while a jet was bouncing, the bath velocity at which the jet stopped bouncing was lower than the bath velocity at which the bouncing jet could be initiated. The second sense of hysteresis is that a jet impinging on a moving bath has up to four distinct states that occur for the same experimental conditions.

\section{Multiple stable states}

Three states that are stable for the same conditions are shown in Fig. 6. Each state can be changed to another state by passing a plastic rod through the falling stream.

Figure 6(a) shows a thin cylindrical film of air being entrained into the bath by the impinging jet. (An impinging jet in a stationary bath was studied by Lorenceau et al. [2,25].) The horizontal motion of the bath drags along the jet and the air film. Air is entrained continuously and collects at the end of the sheath; occasionally a bubble pinches off. The unstable air sheath also formed antibubbles. This occurred more frequently at our lowest viscosity value $(52 \mathrm{mPa} \mathrm{s})$, consistent with previous studies [26,27]. When the antibubbles breached the surface, they became large drops of oil floating on top of the bath surface [28].

A second state, which we call the "half-entraining jet," is shown in Fig. 6(b). Only the bottom edge of the jet entrains air; the top edge of the jet is deep in the bath but does not entrain air, as indicated by the inset of the schematic diagram. As the entrained air collects, small bubbles separate from the bottom edge of the air sheath.

Another confirmation where an air layer separates the jet and the bath is given by increases in the bath velocity, which lengthen the air sheath of the half-entraining jet into a nearly semicircular arc until the jet rises above the bath level and floats on the surface. Since the rotation rate could be adjusted continuously, this transition could be approached slowly. Once the jet is trailing on the surface, decreasing the bath velocity can lead to the jet lifting from the bath surface; this was mentioned previously as the third method to start a jet bouncing.

The bouncing jet in Fig. 6(c) is the third state for the same conditions. The jet deforms the bath's surface for a distance $S$. The geometry is difficult to deduce from the photograph 
because of the refraction, but it is illustrated in the inset of the schematic diagram. As $V_{\text {bath }}$ increased, the jet plunged less deeply below the free surface. The maximum depth that the jet penetrated into the bath decreased linearly as the bath's velocity $V_{\text {bath }}$ was increased from 0.7 to $9.43 \mathrm{~cm} / \mathrm{s}$, while the horizontal distance that the jet traveled below the bath level increased (observations for $Q=0.16-0.52 \mathrm{~cm}^{3} / \mathrm{s}$, $\mu=349 \mathrm{mPa} \mathrm{s}, H=4.2 \mathrm{~cm})$. As $V_{\text {bath }}$ increased, the decrease in the penetration depth was greater than the increase in the horizontal distance, so that the length scale $S$ of the bouncing jet decreased slowly.

In a fourth state, observed for some conditions but not those in Fig. 6, the jet merged with the bath upon contact with the bath, causing only a small depression of the bath surface around the circumference of the jet. The conditions for merging smoothly and for entraining air were discussed in $[1]$.

\section{ENERGY OF PLUNGING AND BOUNCING JETS}

We suggest that the transition from plunging to bouncing, illustrated in Fig. 6 and marked by the lower curves in Fig. 5 (a), corresponds to a competition between the work associated with dragging the plunging jet through the bath and the energy needed to create the additional bath surface when the jet is bouncing. Effects such as buoyancy, inertia, and shear in the air film are important in the initiation and process of bouncing but are assumed to be unimportant for the argument.

Consider the energy associated with the additional surface that the rebounding jet makes compared to a nonentraining plunging jet. The energy of the new interfacial surface scales as an area

$$
E_{\text {bouncing }}=\int \sigma d A \sim \sigma S^{2},
$$

where $S$ is the arclength of the roughly semiellipsoidal indentation on the bath. The surface area present before bouncing changes only the coefficient, not the scaling. Additionally, the length scale $S$ scales linearly with $Q$ with a slope $b=3.13 \mathrm{~s} / \mathrm{cm}^{2}$, as shown in the inset of Fig. 7. Equation (1) then becomes $E_{\text {bouncing }} \sim \sigma b^{2} Q^{2}$.

Now consider the energy of the jet plunging into the bath. The jet slows down, widens, and is carried along by the bath; the trumpet-shaped air film can be seen in Fig. 6(a). We model the plunging jet simply as a straight vertical cylinder of length $L$ with a diameter $d_{\text {jet }}$ moving through a fluid at velocity $V_{\text {bath }}$ perpendicular to the cylinder's axis. This very rough model is used just to obtain some indication of parameter dependencies. The length $L$ is some effective length over which the plunging jet penetrates into the bath; it is not necessarily the length of the air sheath. The Stokes drag force $F_{d r a g}$ exerted on the cylinder is

$$
F_{\text {drag }}=\frac{4 \pi \mu V_{\text {bath }} L}{\ln \left(7.4 / \operatorname{Re}_{\text {bath }}\right)},
$$

where $\operatorname{Re}_{\text {bath }}=d_{\text {jet }} \rho V_{\text {bath }} / \mu$ [29], calculated using $d_{\text {jet }}$ $=\sqrt{Q} \sqrt{4 /\left(\pi V_{\text {jet }}\right)}$, ranged from 0.01 to 6 .

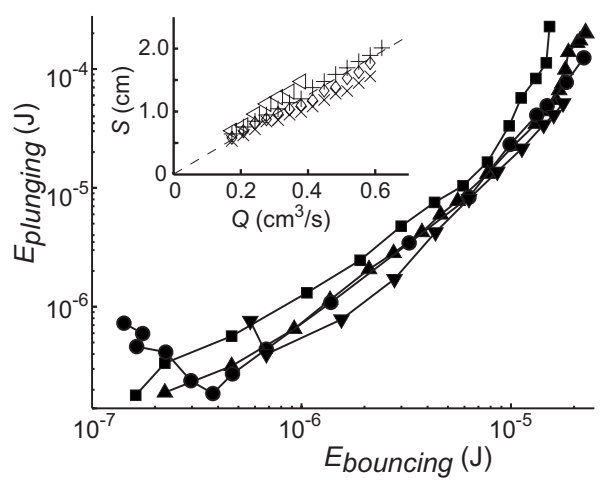

FIG. 7. Plunging is argued to be energetically favored below the curves while bouncing is energetically favored above the curves (see text). The data for four viscosity values (ranging by a factor of 7) collapse onto the same approximate curve. The energy $E_{\text {plunging, }}$, given in (5) using the length $L$ of (7), is from the viscous drag of the plunging jet. The energy $E_{\text {bouncing }}$, given in (1), is from the surface area of the bouncing jet. The solid symbols and data points are the same as in Fig. 5(a). Inset: the penetration length $S$ [see Fig. 6(c)] varies linearly with $Q(\mu=211 \mathrm{mPa}$ s) for different bath velocities $V_{\text {bath }}=7.0 \mathrm{~cm} / \mathrm{s}(\triangleleft), 14.0 \mathrm{~cm} / \mathrm{s}(+), 17.5 \mathrm{~cm} / \mathrm{s}(\diamond), 21.0 \mathrm{~cm} / \mathrm{s}$ $(\times)$.

The associated work is

$$
E_{\text {plunging }}=\int F_{d r a g} d x \propto F_{d r a g} \ell,
$$

where $\ell$ is the length of integration. The length $\ell$ is taken as the horizontal distance that the bath advects a parcel of the jet during the time $\Delta t$ that it traverses the cylinder of length $L$ traveling with velocity $V_{\text {jet }}$,

$$
\ell=V_{\text {bath }} \Delta t=V_{\text {bath }} \frac{L}{V_{\text {jet }}} .
$$

Substituting $\ell$ into the expression for the energy of plunging yields

$$
E_{\text {plunging }} \sim \frac{4 \pi \mu V_{\text {bath }}^{2} L^{2}}{V_{\text {jet }} \ln \left(7.4 / \operatorname{Re}_{\text {bath }}\right)} .
$$

In our model, the energies of the drag and of the new surface are balanced at the transition between plunging and bouncing,

$$
\frac{4 \pi \mu V_{\text {bath }}^{2} L^{2}}{V_{\text {jet }} \ln \left(7.4 / \mathrm{Re}_{\text {bath }}\right)} \sim \sigma b^{2} Q^{2}
$$

The length $L$ was not measured experimentally, but it is expected to increase with $Q$ and $V_{j e t}$, and decrease with $\mu$ and $V_{\text {bath }}$. We take as an ansatz

$$
L \sim a \sqrt{\frac{Q}{V_{\text {bath }}}},
$$

where $a=20$ (dimensionless) makes the length comparable to estimates of the effective jet length, which is longer than the air sheath (typically $0.5-2 \mathrm{~cm}$ ) since the jet penetrates farther than the air sheath. With the factor $a$ the energies $E_{\text {plunging }}$ 
and $E_{\text {bouncing }}$ are comparable, but given the neglected coefficients and many rough approximations, the energy magnitudes are very uncertain. Because most of the jet's velocity is due to gravity and the data are taken at the same height $H$, we assume $V_{j e t}$ to be constant. Our measurements of the transition curves at four different viscosity values collapse well when expressed as in (6) with ansatz (7), as Fig. 7 illustrates. A power law fit of $E_{\text {bouncing }}$ and $E_{\text {plunging }}$ (for the region where the curves have positive slope) yields an exponent of 1.6.

The energy balance argument collapses the data (Fig. 7), but other mechanisms could determine the transition in some range of parameters. For example, one condition for a steadily bouncing jet is that the flux of air coming into the air layer must balance the air leaving it; however, without knowing the air sheath thickness we cannot test this idea.

We also considered alternatives to our ansatz for the length (7). For example, one can solve (6) from the energy balance argument,

$$
L^{\prime} \sim \frac{b Q}{2 V_{\text {bath }}} \sqrt{\frac{\sigma V_{\text {jet }}}{\mu \pi} \ln \left(\frac{7.4}{\mathrm{Re}_{\text {bath }}}\right)} .
$$

A second alternative length,

$$
L^{\prime \prime} A \sim \frac{\rho Q}{4 \pi \mu} \frac{V_{\text {jet }}}{V_{\text {bath }}},
$$

can be obtained by balancing the kinetic energy of the falling jet with the viscous drag on the jet as it plunges axially into a bath. The kinetic energy is $E_{\text {falling }}=\frac{1}{2} \rho Q V_{\text {jet }}^{2} t$, where $t$ is a characteristic time. The energy of drag on an axially moving cylinder is $E_{\text {axial }}=L^{\prime \prime} F_{\text {axial }}=2 \pi \mu L^{\prime \prime 2} V_{\text {jet }} A$, where $A=\alpha /$ $(1-0.81 \alpha)$ and $\alpha^{-1}=\ln \left(2 L^{\prime \prime} / d_{j e t}\right)$ [30]. Using a characteristic time $L^{\prime \prime} / V_{\text {bath }}$ and solving for $L^{\prime \prime}$ yields (9).

The three lengths $L, L^{\prime}, L^{\prime \prime}$ all increase with $Q$ and decrease with $V_{b a t h}$, as expected, but they also diverge for $V_{\text {bath }} \rightarrow 0$, which is unphysical. It is unclear which length better describes the physical situation, but only the ansatz collapses the transition curves (Fig. 7).

\section{KITCHEN EXPERIMENTS}

The bouncing jet phenomenon can be observed in many household fluids such as canola oil or heavy mineral oil. Bouncing was first observed in our laboratory while pouring silicone oil by hand into a dish for storage. The materials needed for observing a bouncing jet are simple: a dish (preferably transparent like a glass pie pan, at least $15 \mathrm{~cm}$ in diameter and $4 \mathrm{~cm}$ tall), a cup, and a small rod (e.g., a cable tie or a chopstick). We measured the viscosity of canola oil and heavy mineral oil (at $22{ }^{\circ} \mathrm{C}$ ) at high shear rates and found both oils to be Newtonian to a good approximation: for canola oil, $\mu=65 \mathrm{mPa}$ s at low shear and $4 \%$ lower at a shear of $10^{4} \mathrm{~s}^{-1}$; for heavy mineral oil, $\mu=180 \mathrm{mPa}$ s at low shear and $18 \%$ lower at a shear rate of $10^{4} \mathrm{~s}^{-1}$.

To observe a bouncing jet, use a dish with liquid about 4 $\mathrm{cm}$ deep and pour a thin stream of the liquid $\left(0.5-1 \mathrm{~cm}^{3} / \mathrm{s}\right)$ from a cup $3-6 \mathrm{~cm}$ above the surface. While pouring, move the stream in a circular motion around the dish once about every $2 \mathrm{~s}$ at a distance $3-6 \mathrm{~cm}$ from the center. Watch for the jet to bounce while varying the pouring rate, the relative horizontal velocity between the jet and the bath, and the pouring height. To encourage bouncing, pass the small rod through the jet intermittently. A rotating platform (e.g., a record turntable or a lazy susan) can be used to rotate the dish instead of moving the cup. If the surface is dirty, clean the surface by stirring the bath or scraping the surface $[11,22]$. Blow air on the surface to pop bubbles on the surface. To achieve a very small pouring height, pour the liquid down the rod.

With practice, a jet poured by hand can bounce stably for tens of seconds at a time. For more detailed instructions and for more liquids and conditions, see [28]. Bouncing is also easy to observe in non-Newtonian fluids such as shampoo, multigrade motor oil, and concentrated mixtures of liquid soap and water, but the mechanism by which they bounce may not be the same as for Newtonian fluids.

\section{DISCUSSION}

We have observed a falling jet of a Newtonian liquid bouncing from a moving bath of the same liquid for a wide range of viscosity $(52-349 \mathrm{mPa} \mathrm{s})$, jet diameter $(0.05$ $-0.12 \mathrm{~cm})$, jet velocity at impact $(38-170 \mathrm{~cm} / \mathrm{s})$, and the bath's horizontal velocity $(0.5-50 \mathrm{~cm} / \mathrm{s})$. By initiating the jet in different ways, as many as four stable states were observed for the same experimental conditions.

The bouncing jet is an additional example of steady noncoalescence. Bouncing jets could be used as a technique for controlling a fluid jet and preventing or promoting the entrainment of the fluid surrounding the jet. The phenomenon can be observed easily with a variety of fluids at home.

The bouncing phenomenon we have studied is similar to the Kaye effect: both are thin streams of liquid rebounding from a surface, and both occur for similar falling heights, jet velocities, and jet diameters. However, the liquid of the stable Kaye effect is non-Newtonian, while we have studied bouncing for Newtonian liquids. The Newtonian bouncing liquid jet is separated from the bath by an air layer, likely 0.1 to $10 \mu \mathrm{m}$ thick (see discussion in Sec. III A) $[2,17,24]$. In contrast, the stable Kaye effect is lubricated by a shearthinned layer of liquid about $100 \mu \mathrm{m}$ thick [21]. The bouncing jet also occurs for less viscous liquids than the Kaye effect.

The behavior of a bouncing jet is determined by an interplay of viscous, inertial, surface, and gravitational forces. This causes the relationship between any two features of the bouncing jet to be very complicated. We have suggested that the onset of bouncing can be understood by comparing the energy of drag on the plunging jet with the energy of the new surface of the bouncing jet. While the argument can collapse the transition curves, it cannot explain the slope of the curves or their closure at small $Q$. To understand the bouncing transition better, future experiments should measure the jet length $L$ and the thickness of the air sheath, and examine the bouncing jet's dependence on surface tension, density difference, radius of the nozzle, angle of incidence of the jet, and 
the viscosity and pressure of the surrounding fluid (which was air at atmospheric pressure in our experiments). Most of these conditions have already been studied for the case of droplet noncoalescence $[5,17]$.

We have also observed that a falling jet impinging on a moving bath exhibits many other phenomena that warrant future study [28].

\section{ACKNOWLEDGMENTS}

We thank W. D. McCormick and J. W. M. Bush for helpful discussions. We also thank Olivier Praud and Abe Yarbrough for help with preliminary experiments. Acknowledgment is made to the Donors of the American Chemical Society Petroleum Research Fund for support of this research.
[1] J. Eggers, Phys. Rev. Lett. 86, 4290 (2001).

[2] E. Lorenceau, D. Quéré, and J. Eggers, Phys. Rev. Lett. 93, 254501 (2004).

[3] M. Maleki, M. Habibi, R. Golestanian, N. M. Ribe, and D. Bonn, Phys. Rev. Lett. 93, 214502 (2004).

[4] Y. Couder, E. Fort, C.-H. Gautier, and A. Boudaoud, Phys. Rev. Lett. 94, 177801 (2005).

[5] G. P. Neitzel and P. Dell'Aversana, Annu. Rev. Fluid Mech. 34, 267 (2002).

[6] K.-Y. Lee, H.-G. Lee, and P. C. Hayes, ISIJ Int. 38, 1233 (1998).

[7] P. Dell'Aversana, V. Tontodonato, and L. Carotenuto, Phys. Fluids 9, 2475 (1997).

[8] A. K. Biń, Chem. Eng. Sci. 48, 3585 (1993).

[9] P. G. Simpkins and V. J. Kuck, J. Colloid Interface Sci. 263, 562 (2003).

[10] Lord Rayleigh, Proc. R. Soc. London 28, 405 (1878-1879)..

[11] O. Reynolds, Proc. Manchester Lit. Philos. Soc. 21, 1 (18811882).

[12] J. Walker, Sci. Am. 238, 151 (1978).

[13] A. L. Yarin, Annu. Rev. Fluid Mech. 38, 159 (2006).

[14] Y. Amarouchene, G. Cristobal, and H. Kellay, Phys. Rev. Lett. 87, 206104 (2001).

[15] N. Vandewalle, D. Terwagne, K. Mulleners, T. Gilet, and S. Dorbolo, Phys. Fluids 18, 091106 (2006).

[16] G. P. Neitzel, P. Dell'Aversana, and D. Castagnolo, in Proceedings of the Fourth Microgravity Fluid Physics and Transport Phenomena Conference, NASA Lewis Research Center, Cleve- land, OH, 1999 (unpublished), Document ID: 20010004358.

[17] M. R. Vetrano and P. Dell'Aversana, Experiments on AirLubricated Liquids: Role of Vapor, Electrostatic Effects, and Heat Transmission across the Interface, Lecture Notes in Physics Vol. 628 (Springer, Berlin, 2003), online-only supplement.

[18] L. D. Mahajan, Philos. Mag. 10, 383 (1930).

[19] J. Ferguson, N. E. Hudson, and B. C. H. Warren, J. NonNewtonian Fluid Mech. 23, 49 (1987).

[20] A. Kaye, Nature (London) 197, 1001 (1963).

[21] M. Versluis, C. B. D. van der Meer, K. van der Weele, and D. Lohse, J. Stat. Mech.: Theory Exp. (2006), P07007.

[22] Lord Rayleigh, Philos. Mag. 48, 321 (1899).

[23] M. Thrasher, S. Jung, Y. K. Pang, and H. L. Swinney, Phys. Fluids 19, 091110 (2007).

[24] O. W. Jayaratne and B. J. Mason, Proc. R. Soc. London, Ser. A 280, 545 (1964).

[25] E. Lorenceau, F. Restagno, and D. Quéré, Phys. Rev. Lett. 90, 184501 (2003).

[26] S. Dorbolo, H. Caps, and N. Vandewalle, New J. Phys. 5, 161 (2003).

[27] S. Dorbolo, E. Reysaat, N. Vandewalle, and D. Quéré, Europhys. Lett. 69, 966 (2005).

[28] M. Thrasher, MA thesis, University of Texas at Austin, 2005; $\mathrm{PhD}$ dissertation, University of Texas at Austin, 2007.

[29] H. Lamb, Hydrodynamics, 6th ed. (Cambridge University Press, Cambridge, U.K., 1932).

[30] R. Cox, J. Fluid Mech. 44, 791 (1970). 